\title{
Stability of the dynamic shifting process in the vehicle transmission with the input shaper
}

\author{
Alexander Taratorkin ${ }^{1}$, Victor Derzhanskii ${ }^{1,2}$, Igor Taratorkin ${ }^{1, *}$ \\ ${ }^{1}$ IES UB RAS, Department of Transportation Vehicles Mechanics, 620049, Ekaterinburg, Russia \\ ${ }^{2}$ Kurgan State University, Department of Applied Mechanics, 640020, Kurgan, Russia
}

\begin{abstract}
This paper investigates stability of the dynamic process of gear shift in the vehicle transmission with the input shapers (IS) taking in consideration uncertainty of the natural frequency of the mechanical system. The proposed control algorithm increases the stability of the researched system with variation of its elastic and inertial parameters. The control law with guaranteed asymptotic stability is obtained for full load of the vehicle when the all-wheel drive is turned on. The monitoring of the state and performance of the required parameters for the regulation of input shapers are tested by means of numerical simulation. Analyzing the results it is established that the best stability is reached by the adaptive setting of the input shapers in accordance with controlled value of the natural frequency of the lowest single-node mode.
\end{abstract}

\section{Introduction}

When evaluating vehicle dynamic loading, it is established that low-frequency torsion oscillations in the transmission, corresponding to the first one-node Eigen mode, are observed in the transient processes of starting from stand still and gear shifting. The power of these oscillations is sufficient to excite the longitudinal oscillations of the vehicle body (up to $2 \ldots 4 \mathrm{~m} / \mathrm{s} 2$ ). In many cases, known solutions for exclusion this torsion oscillations do not provide the required quality of the dynamic processes that arise after locking friction elements of the shifted gear (after shaping a new structure of the dynamic system). As a rule, these methods resolve themselves to regulating the pressure of the power fluid in the actuating cylinders of friction elements [1]. This leads to a decrease in the vehicle performance properties. Therefore, the problem of the scientific substantiation and development of new algorithms for controlling transient processes in the power-train unit of the vehicle after locking friction elements of the shifted gear is actual.

The goal of the research is to substantiate the method of damping undesirable lowfrequency oscillations in the transmission after the gear shift during vehicle boost acceleration.

The scope of the research is:

\footnotetext{
${ }^{*}$ Corresponding author: ig_tar@mail.ru
} 
- substantiation of the method for damping low-frequency oscillations in the transport vehicle transmission based on applying an algorithm for power control input redistribution during a time period;

- evaluation of effectiveness of the applied algorithm for redistribution of the power control input during the time period of gear shifting in the transport vehicle transmission and determination of restrictions on its application.

The object of theoretical and experimental research was an all-wheel drive vehicle, $3,000 \mathrm{~kg}$ in weight with a hybrid power-train unit and automatic transmission.

In the course of the experiment, the kinematic and power parameters that characterize the dynamics of the powertrain unit as well as the vehicle were measured.

From the results of the experiment, it follows that in the process of shifting gears up from the $1^{\text {st }}$ to the $5^{\text {th }}$, low-frequency oscillations are excited on the transmission shafts, the amplitude of which reaches significant values. The oscillation frequencies depend on the number of the shifted gear and they are located in the range from 1.5 to $7.1 \mathrm{~Hz}$. In this case, the values of the longitudinal acceleration of the vehicle body reach $2 \ldots 4 \mathrm{~m} / \mathrm{s}^{2}$. It should be noted that the parameters of the transient process in the system under consideration can be influenced significantly by a variation in the natural frequency of the system at certain gears in the transmission. Such a change in the natural frequency can also happen due to nonlinearity of the system, due to vehicle mass variation because of different degrees of load capacity utilization, of fuel reserve, due to tire temperature and pressure, etc. Thus, the effect of the natural frequency variation is taken into consideration, when developing the vibration damping algorithm, and it is estimated by robustness of the control system algorithm.

With reference to the problem of studying transient processes of starting from standstill and shifting gears, the control action can be considered as a single function, since when the vehicle is accelerated after equalizing the angular speeds of the control elements, the required specific driving force which provides for vehicle boost acceleration with the required acceleration is determined by the following formula:

$$
f_{d}=\frac{\dot{v}}{g \cdot \delta_{j}}+f_{r}
$$

where, $f_{d}, f_{r}$ is a specific driving force and resistance to movement, respectively; vis the required boost acceleration; $g$ is gravitational acceleration $\left(g=9.81 \mathrm{~m} / \mathrm{s}^{2}\right) ; \quad \delta_{j}$ is the rotational inertia coefficient at the $\mathrm{j}^{\text {th }}$ gear. Thus, when the vehicle is accelerated at the first gear with an acceleration of $2.5 \mathrm{~m} / \mathrm{s}^{2}$, the ratio $f_{d} / f_{r}=0.02=9.45$, i.e. the required control action exceeds the rolling resistance torque by an order of magnitude. The system response to the given control action can be considered as a response to a single control function.

\section{Substantiation of the method of damping low-frequency oscillations in the vehicle transmission}

It is a fact that in studying torsional oscillations in the transport vehicle transmissions corresponding to the first oscillations Eigen mode in steady and transient modes of motion, it is well-founded to apply two-mass mathematical models [12] that adequately simulate the real process. The corresponding Eigen mode has one knot of oscillations located in the "semiaxis - tire" zone). The natural frequency and the exact position of the oscillation node are determined by the number of the shifted gear. This Eigen mode corresponds to a two- 
mass oscillator, the simulation model of which was used below to evaluate the effectiveness of the proposed power redistribution of control actions.

This model allows for investigating the so-called Bonanza effect, excitation of lowfrequency oscillations when starting the engine, shifting gears and accelerating the vehicle. When starting the vehicle engine and accelerating the vehicle, sharp exertion of the control torque is carried out. Due to the absence of attenuation, the transmission can vibrate for a long time. The amplitude of the oscillation moment is in the range between zero and twice the value of the control torque. This is the Bonanza effect. At the same time, vibrations are perceived as disturbing because they increase the load on the transmission components and decrease the travelling comfortableness. Devaluation of this effect can be achieved by introducing effective damping or by system detuning, i.e. by transferring the natural frequency to the bandwidth that is not critical for passengers and transmission elements. Unfortunately, both solutions are difficult to implement in practice due to significant configuration and engineering design constraints. Alternatively, the pulse which causes vibration (applied torque) can be changed to prevent or reduce the excitation of free oscillations.

The approach set forth in work [1] is based on the idea of damping oscillations by forming corrective pulses in antiphase to the oscillation process, i.e. by power redistribution of the control action taking into account dynamic system frequency characteristics. This ideology is implemented in the method of so-called input shapers [2-4] and has found wide use in various industrial applications. The main idea of Input Shapers comprises formation of a controlling signal by convolution of the controlling input with the pulse sequence in the form of the Dirac delta function. Shaper algorithm is based on redistribution of power impact on a controlled object in the course of time, while preserving an invariable value of the total impact. Due to a relative increase in time of system transient from one state into another, compensation of excited oscillations is provided, and in an ideal case, the shift of the control action for a fraction of the period of the calculated oscillations of the controlled object provides their full suppression.

It should be considered that real-life controlled objects (in particular, the gear shifting control system of the vehicle) are non-linear, from the one hand, and from the other, their frequency characteristics significantly depend on the number of the shifted gear. This leads to variations of the specified parameters. Thus, there appears a task of shaping such an input signal which will provide robustness in the system in relation to inconsistency of excited oscillation frequency (natural frequency) and to the controlled object damping coefficient[5].

Another important property characterizing the quality of Input Shapers performance is high-speed characteristics which is defined by the time of the latest pulse $t_{n}$. For this reason, when calculating the algorithm, a condition of $t_{n}$ minimization is usually entered. In such a case, it is necessary to introduce restrictions for pulse minimization amplitude as far as time minimization can result in the necessity of shaping pulses with an infinite amplitude. Execution of the given conditions is defined by the dynamics of the object under research and by the choice of a Shaper-algorithm type.

Input Shapers have found a wide-spread occurrence in different industrial applications [6] that allowed for increasing considerably the performance of various equipment such as of cranes $[7,8]$, coordinate measuring machines [9,10], satellite systems [11], milling machines [12], disk drives [20], transport vehicle motion control system $[13,14]$ and others. The above-mentioned references describe the types of Shapers, dependences on their parameter determination, efficiency assessment for different industrial applications and recommendations on their use.

However, with reference to transport vehicles, such control algorithms that can potentially provide for high-speed characteristics, the required level of residual oscillations and 
robustness have not been properly applied. To assess the possibility of using this wellformalized method to improve the quality of the transient after the gear shift, a mathematical model of the mechanical system is designed, the values of the natural frequencies and the parameters of the power redistribution function are determined. Simulation modelling was performed in LMS Imagine.Lab AMESim.

According to the modelling results, it is established that the oscillation frequency varies in the range from 1.6 to $7.1 \mathrm{~Hz}$. The results of numerical modelling for the initial scheme with various variants of power redistribution of the control action are shown in Figure 1.

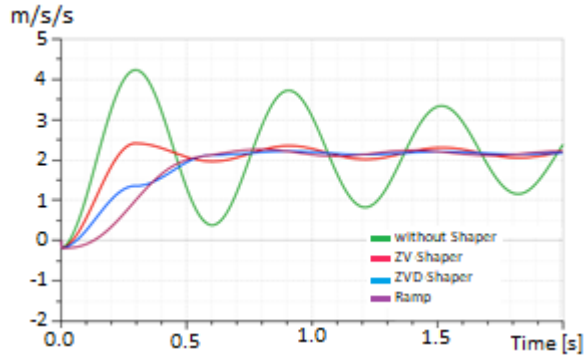

Figure 1. Graphical representation of the parameters characterizing the dynamics of the investigated processes during the vehicle acceleration after shifting the first gear.

As follows from the Figure, in the absence of power redistribution of the control action, the overshoot is $91 \%$, the response rate is $0.15 \mathrm{sec}$. Introduction of ZV-Shaper improves the quality of the transient process (overshoot - 9,1\%, the response rate is $0,24 \mathrm{sec}$ ). Introduction of ZVD-Shaper demonstrates good response rate $0.6 \mathrm{sec}$., at this, in the first half-period of an oscillation (up to the value 0.65 of the required torque), response rate is comparable to the ZV-Shaper. At this, overshoot is $0.9 \%$. Damping of excited oscillations after shifting the gear when the control torque increases according to the RAMP law is characterized by a $2.6 \%$ overshoot at the first gear and high speed characteristics $0.6 \mathrm{sec}$, as with the ZVD-Shaper. Dependencies for various control laws for the values of overshoot and response rate on the natural frequency of the system under consideration (the numbers of the shifted gear) are illustrated in Figure 2 (A, B).

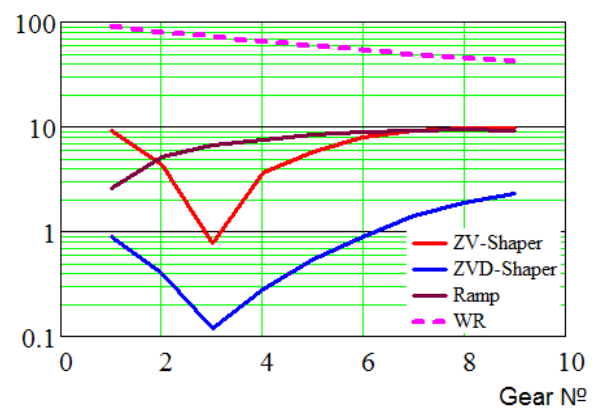

A) Overshoot, \%

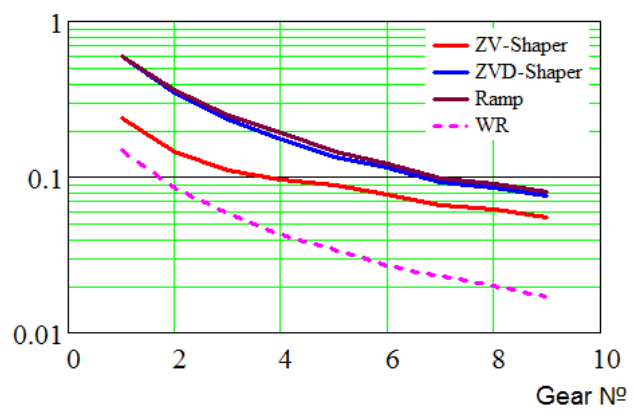

B)Response rate, $\mathrm{s}$

Figure 2 - Distribution nature of overshoot and response rate values for various control laws, depending on the number of the gear shifted (on the system natural frequency)

As follows from Figure 2, the RAMP control law demonstrates a sequential increase in overshoot from $2.6 \%$ to $9.3 \%$ with an increase in the natural frequency from 1.6 to $7.1 \mathrm{~Hz}$. The control algorithm for the ZV-Shaper and ZVD-Shaper provides a minimum overshoot at the $3^{\text {rd }}-4^{\text {th }}$ gear (the frequency is $3.3-4.2 \mathrm{~Hz}$.). Outside this bandwidth, overshoot increases, which can be explained by a greater degree of dynamic system stability in a given frequency range. 
Another parameter for evaluating Shaper algorithms effectiveness is robustification at a given level of residual oscillations. The results of the performed analysis according to function (3) for the pre-determined amplitudes of the control action in accordance with the Shaper algorithms under consideration are shown in Figure 3.

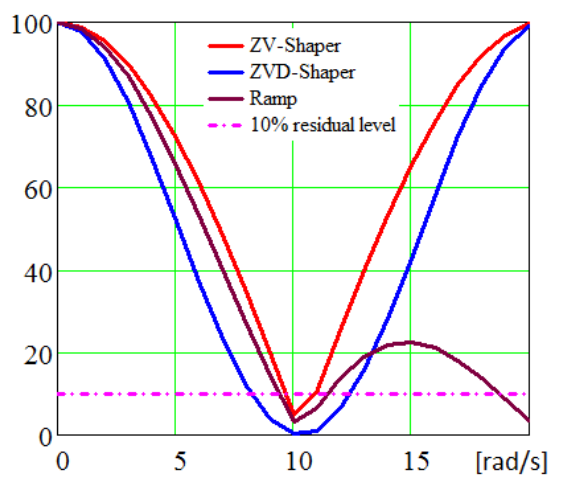

Table 1. Performance evaluation of the algorithms under study.

\begin{tabular}{|c|c|c|c|}
\hline $\begin{array}{l}\text { Input } \\
\text { Shapers }\end{array}$ & $\begin{array}{l}\text { Response } \\
\text { Rate }\end{array}$ & Overshoot & Robustness \\
\hline $\begin{array}{c}\text { ZV- } \\
\text { Shaper }\end{array}$ & 1 & 3 & 3 \\
\hline $\begin{array}{c}\text { ZVD- } \\
\text { Shaper }\end{array}$ & $2-3$ & 1 & 1 \\
\hline RAMP & $2-3$ & 2 & 2 \\
\hline
\end{tabular}

Figure 3. Evaluation of various Input Shaper robustness for a given level of residual oscillations, $\%$

The results of a joint analysis of the overshoot, response rate and robustness of various Input Shapers allows for making a conclusion about the ambiguity of evaluating certain parameters. For example, the best performance is demonstrated by the ZV-Shaper.

Besides, it happens to be the worst from the point of view of robustness, i.e. with the natural frequency variation, the algorithm efficiency is significantly reduced. Comparable with ZV-Shaper, the value of the robustness magnitude is demonstrated by the RAMPalgorithm, but its performance is equal to the corresponding ZVD-Shaper and is significantly inferior to the ZV-Shaper. At the same time, ZVD-Shaper demonstrates the best overshoot values, ZV-Shaper - the worst, and RAMP-algorithm is comparable with it. For visual assessment of the effectiveness of various algorithms, the estimated parameters of overshoot, response rate and robustness are summarized in Table 1, indicating the degree of significance for particular algorithms.

From the quoted results, it follows that none of the algorithms considered provides the best quality for all three parameters. The choice of the algorithm type can be carried out depending on the priority of the quality parameter of the transient process. At the same time, it follows from the results of modelling the dynamics of the power train unit of the object under study that it is expediential to use the ZVD-Shaper for the first three gears. If you require better response rate, you can use the ZV-Shaper. For this, it is necessary to investigate a possibility of including an additional PID controller in the control circuit. For gears starting from the $4^{\text {th }}$ one and higher on, it is advisable to use a RAMP-algorithm, which is the simplest to be implemented from the technical point of view. In this case, the control action rise time should correspond to the period of natural frequency for the given gear. Application of the control action for a time shorter than the period duration leads to a sharp deterioration of the overshoot and robustness. Application of the control action for the time exceeding the duration period of natural oscillations leads to an unacceptable decrease in boost acceleration dynamics.

\section{Conclusions}

As a result of the performed computational and experimental studies of the dynamics of the controlled vehicle motion during boost acceleration, it is established that the level of 
transmission dynamic loading and comfortableness are limited by the quality of response to the control action during vehicle boost acceleration after shifting the gear. In order to achieve the required quality of transient control after locking the friction elements of the gear being shifted, it is expedient to apply algorithms for redistribution of the power control action, which ensures a satisfactory correlation among inconsistent response rate, the degree of oscillation damping and robustness. With reference to the object under study, it is advisable to implement the ZVD-Shaper algorithm for controlling transients at the $1^{\text {st }}-2^{\text {nd }}-$ $3^{\text {rd }}$ gears. Maintenance of the required transients quality at the remaining gears can be achieved with a linear rise of the control action for a time period corresponding to the period of natural frequency of the first oscillation mode of the dynamic system at the shifted gear (RAMP algorithm).

This study was funded by the program of the President of the Russian Federation for the support of young scientists - Grant MK-5809.2018.8, and also with partial support under the state task IES UB RAS No. 0391 - 2014 - 007.

\section{References}

[1] R. Fischer, F. Küçükay, G. Jürgens, R. Najork, B. Pollak, The Automotive Transmission Book, ISBN 978-3-319-05262-5, ISBN 978-3-319-05263-2 (eBook) DOI 10.1007/978-3-319-05263-2, (2015)

[2] W. E. Singhose, E. A. Crain and W. P. Seering, Convolved and Simultaneous TwoMode Input Shapers. IEE Control Theory and Applications 11 515-520 (1997)

[3] H. Kojima,W. Singhose, Adaptive Deflection Limiting Control for Slewing Flexible Space Structures, Journal of Guidance, Control and Dynamics 30 61-67 (2007)

[4] C. F. Cutforth, L. Y. Pao, Analysis and Design of an Adaptive Input Shaper for the Control of Flexible Structures, Proceedings of the American Control Conference, Anchorage AK, AACC, p. 1903-1910(2002).

[5] N. C. Singer, W. P. Seering, Dynamic Systems, Measurement, and Control 112, P. 76$82(1990)$

[6] K. L. Sorensen, Operational performance enhancement of human operated flexible systems. Georgia Institute of Technology(2008)

[7] K. L. Sorensen, W. E. Singhose, S. Dickerson, Control Engineering Practice,15, p. 825-837 (2007)

[8] A. P. Kuznetsov, A.V. Markov, A. S. Shmarlouski, T. V. Gavrilik, Shaping algorithms enabling to reduce vibration of control objects. p. 5-12 (2011)

[9] N. Seth, K. Rattan, R. Brandstetter, IEEE Conf. on Control Applications.. p. 368373(1993)

[10] S. Jones, A.G. Ulsoy, Dynamic Systems, Measurement and Control,121, p. 242-247 (1999)

[11] W. E. Singhose, L. J. Porter, T.D. Tuttle et al., Dynamic Systems, Measurement and Control.. 119, p. 320-326 (1997)

[12] J. Fortgang, W. Singhose, J. Marquez. et al., American Control Conf. Proceeding, p. 4531-4536(2005)

[13] W. E. Singhose, W. P. Seering, N. C. Singer, Dynamic Systems, Measurement and Control,119, p. 198-205(1997)

[14] I. Taratorkin, Improving the quality of transient response during automatic control of the turn of a tracked vehicle based on the implementation of structured input shapers / Igor Taratorkin, Victor Derzhanskii and Alexander Taratorkin. MATEC Web of Conferences 129, 06029 (2017) DOI: 10.1051/matecconf/2017129, 06029 ICMTMTE (2017) 